\title{
3 Research Square

\section{Observational and genetic evidence on the association of sleep behaviors with the incidence of fracture}

\author{
Yu Qian \\ Westlake University \\ Xia Jiangwei \\ Westlake University \\ Ke-Qi Liu \\ Jiangxi Medical College \\ Lin Xu \\ Binzhou Medical University \\ Shu-Yang Xie \\ Binzhou Medical University
}

\section{Guo-Bo Chen}

People's Hospital of Hangzhou Medical College

\section{Pei-kuan cong}

Westlake University

Khederzadeh Saber

Westlake University

Houfeng Zheng ( $\square$ zhenghoufeng@westlake.edu.cn )

Westlake University https://orcid.org/0000-0001-5681-8598

\section{Article}

Keywords: sleep behavior, fracture, genetic evidence

Posted Date: April 30th, 2021

DOl: https://doi.org/10.21203/rs.3.rs-446335/v1

License: (c) (1) This work is licensed under a Creative Commons Attribution 4.0 International License. Read Full License

Version of Record: A version of this preprint was published at Communications Biology on November 26th, 2021. See the published version at https://doi.org/10.1038/s42003-021-02861-0. 


\section{Abstract}

Here, we combined conventional evidence from longitudinal data in UK Biobank and genetic evidence from Mendelian randomization (MR) approach to infer the causality between sleep behaviors and fracture risk. We found that participants with insomnia showed a $7.0 \%$ higher risk of fracture (hazard ratio $\left.[\mathrm{HR}]=1.070,95 \% \mathrm{Cl}=1.045-1.096, P=1.87 \times 10^{-8}\right)$, falls and $\mathrm{BMD}$ mediated $26 \%$ and $10 \%$ of the intermediary effect, the MR analyses provided the consistent evidence. A U-shape relationship was observed between sleep duration and fracture risk $(P<0.001)$ with the lowest fracture risk at sleeping $7-8$ hours per day. The excessive daytime sleepiness and "evening" chronotype were associated with fracture risk in observational study, but the association between chronotype and fracture did not show in MR analyses. We further generated a sleep risk score (SRS) with potential risk factors (i.e., insomnia, sleep duration, chronotype, and daytime sleepiness). We found that the risk of fracture increased with an increasing SRS $\left(\mathrm{HR}=1.095,95 \% \mathrm{Cl}=1.073-1.118, P<2 \times 10^{-16}\right)$. Moreover, $19.4 \%$ of the fracture cases would be removed if participants exhibited a healthy sleep pattern. In conclusion, insomnia had a causal effect on fracture, falls had a larger intermediary effect than BMD in this association. Individuals with fracture risk could benefit from the intervention on unhealthy sleep pattern.

\section{Introduction}

Globally, fracture is a primary cause of disability in adulthood, of which fragility fracture is the major type in the elderly. ${ }^{1}$ In 2000, the estimated number of new osteoporotic fractures worldwide was 9.0 million for people aged 50 years or more, and Europe accounted for the highest number of fractures $(34.8 \%){ }^{2}$ The hip fractures accounted for $18.2 \%$ (1.63 million) of all fractures ${ }^{2}$, and by 2050 , the estimated number of new hip fractures worldwide will increase to 6.26 million cases. ${ }^{3}$ According to the report of epidemiology and burden of osteoporosis in the 27 countries of the European Union (EU27), the health care cost of fragility fractures was estimated to reach $€ 47$ billion for people aged 50 years or more in $2025 .{ }^{4}$ Thus, the primary prevention of osteoporotic fractures is important to reduce the cost burden to society.

Both genetic and environmental factors contribute to the risk of fracture. Several genome-wide association studies have identified dozens of genome-wide significant fracture loci. ${ }^{5-7}$ All loci were associated with bone mineral density (BMD), suggesting that the lower BMD was the major risk factor for fracture. ${ }^{5}$ Besides, environmental factors, such as smoking, ${ }^{8}$ abdominal obesity, ${ }^{9}$ and heavy drinking, ${ }^{10}$ were also found to be associated with increased fracture risk. Recently, increasing evidence from animal studies has put forward the importance of the circadian system and sleep in the process of bone resorption and formation. ${ }^{11,12}$ For example, the clock gene knockout rats, which cannot regulate the circadian rhythms, displayed abnormal bone mass. ${ }^{12}$ Despite these findings, only several observational studies have investigated the relationship between sleep behaviors and fracture risk, but the results were conflicting. For instance, a recent cohort study from the Women's Health Initiative (WHI) observed that short sleep duration, but not long sleep duration, was associated with increased risk of fracture. ${ }^{13}$ However, other studies found that long sleep duration, ${ }^{14}$ self-reported napping, ${ }^{14}$ nocturnal hypoxemia, ${ }^{15}$ 
and premature awakening, ${ }^{16}$ were associated with an increased risk of fracture. Besides, another study reported that insomnia was associated with the risk of falls, but not hip fracture. ${ }^{17}$

While there were some explanations for the controversial findings, unknown confounding factors in an observational study could be one reason. With the public availability of genetic data for sleep traits, the Mendelian randomization (MR) approach, which is a method that applies genetic variants as instrumental variables (IVs) for the exposure of interest, could strengthen the causal inference on the relationship between exposure and outcome (i.e., fracture), ${ }^{18}$ Since the genetic alleles are randomly assorted during conception, MR analyses are less susceptible to confounding factors. ${ }^{18}$ Therefore, in this study, we first conducted a prospective observational study to investigate the relationship of insomnia, sleep duration, excessive daytime sleepiness, snoring, and chronotype with fracture risk within the UK Biobank dataset, then, with the summary GWAS data of fracture, we implemented MR approach to detect the causal relationship between these sleep traits and fracture risk. We finally developed a sleep risk score, which integrates the potential fracture-related sleep traits, and examined the association between sleep risk score and fracture.

\section{Methods}

\section{Study design and data sources}

Detailed information on the study design, data source, and sample data quality control of the UK Biobank has been presented in Supplementary Method, Supplementary Figure 1, and Supplementary Table 1. Briefly, in the observational study and one-sample MR study, we used individual-level data from the UK Biobank (Application 41376) as we used before. 19,20 We excluded 30,487 non-European participants to minimize the population stratification bias. Then, we excluded participants with secondary fractures (i.e., participants with follow-up care involving removal of the fracture plate and other internal fixation devices, pathological fractures, and the fracture of the bone in neoplastic disease), and those with a fracture history, leaving 443,514 participants for the observational study. In one-sample MR analysis, we further excluded 147,330 participants (Field ID 22021) who had a kinship with other participants.

Summary-level data and selection of instrumental variables

Detail information on the selection of instrumental variables has been provided in

Supplementary Method. A total of 268 single nucleotide polymorphisms (SNPs) were 
retained as instrumental variables for chronotype, 184 SNPs for insomnia, 72 SNPs for sleep duration, and 30 SNPs for snoring (Supplementary Table 2 and Supplementary Table 3). Summary-level data for fracture were available from the GWAS that included 53,184 cases and 373,611 controls. ${ }^{6}$ For SNPs that were not available in the outcome data, we used proxy SNPs where available (i.e., genetic variants in LD with the corresponding SNPs at the threshold of $r^{2}>0.9$ ) (Supplementary Table 3).

\section{Assessment of sleep behaviors and definition of sleep risk score}

In the UK Biobank, participants reported five sleep behaviors: insomnia, sleep duration, snoring, daytime sleepiness, and chronotype (Supplementary Table 4). Details of the assessment of sleep behaviors have been provided in Supplementary method. We included the potential fracture-related sleep factors to generate a sleep risk score (SRS). For each high-risk sleep behavior, the participant received a score of 1 if he or she met the criterion for high risk. If the participant did not meet the criterion, he or she was classified at low risk for that behavior, and received a score of 0. SRS was calculated as the sum of all these component scores, with higher scores indicating poor sleep quality.

\section{Assessment of fracture}

In this prospective study, the endpoint was the first diagnosis of any fracture (except secondary fracture). The fracture could be located at the skull and face, neck, vertebrae, ribs, sternum, and thoracic spine, shoulder and upper arm, spine, forearm, wrist, and hand, pelvic and thigh, femur, lower leg, foot except for the ankle, and other unspecified body regions. These cases were included from either questionnaire-based self-reported fractures or hospital-based fractures using the ICD-9 or ICD-10 codes (Supplementary Table 4).

\section{Statistical analyses}

Observational study

In the prospective study of fracture risk, follow-up time was calculated from the date of attending the UK Biobank to the diagnosis of fracture, death, or the censoring date (31 
March 2017). Five sleep behaviors (chronotype, insomnia, snoring, sleep duration, and excessive daytime sleepiness) and sleep risk scores were assessed in relation to the fracture risk using Cox regression. Restricted cubic spline with five knots at $5^{\text {th }}, 35^{\text {th }}, 50^{\text {th }}, 65^{\text {th }}$, and $95^{\text {th }}$ centiles were used to model the potential non-linear association of sleep duration with fracture risk. Detailed information on the multivariable Cox regression models and corresponding covariates is provided in Supplementary Method and Supplementary Table 5. Assuming a causal relationship, we calculated proportional population attributable risk (PAR\%) to estimate the proportion of the fracture incidence in this population that would be eliminated if the exposure were eliminated. We then conducted mediation analyses to estimate the dimensionless proportion of the effect of sleep behaviors on fracture risk mediated by BMD and falls.

To account for multiple comparisons, a Bonferroni-corrected threshold of $P<0.01(0.05 / 5$ sleep behaviors) was considered statistically significant. Findings with $P$-values less than 0.05 but above the threshold of Bonferroni corrected significance were considered to be suggestive evidence. All these statistical analyses were conducted in R version 4.0.2 (http://www.r-project.org/), and the PLINK2.0 software. $^{21}$

\section{One-sample MR study}

In one-sample MR analyses, after extracting SNPs from the UK Biobank imputation dataset (Supplementary Table 3), weighted genetic risk score (wGRS) for four sleep behaviors (chronotype, insomnia, snoring, and sleep duration) were generated using the following equation:

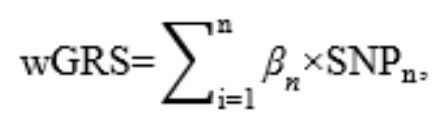

where $\mathrm{SNP}_{\mathrm{n}}$ was the dosage of the effective allele, $\beta_{n}$ was the effect estimate of $\mathrm{SNP}_{\mathrm{n}}$ for each sleep behavior obtained from the previous GWAS, and $n$ was the number of 
instrumental variables ( $\mathrm{n}=268$ for chronotype, 184 for insomnia, 72 for sleep duration, and 31 for snoring) (Supplementary Table 3). The wGRS method was performed using the PLNK2.0 software with the command of --score sum. ${ }^{21}$

After obtaining the sleep behavior wGRS, we performed a multivariable Cox regression to obtain the population average causal hazard ratio using the prospective study of fracture risk, based on model 0 .

\section{Two-sample MR study}

After extracting the genetic association estimates between SNPs and outcomes from summary-level data, ratio estimates for individual SNPs were calculated using the Wald estimator and Delta method. ${ }^{22}$ The inverse-variance-weighted (IVW) method (under random-effects models) was further used to combine these estimates; thus, obtaining the primary causal estimates. ${ }^{22}$ In sensitivity analyses, to assess the influence of potential pleiotropy on the causal effect estimates, the weighted median method, MR Pleiotropy Residual Sum and Outlier (MR-PRESSO) test, and MR-Egger regression were conducted. Specifically, the weighted median method can provide valid estimates if more than $50 \%$ of the weights come from valid instrumental variables. ${ }^{23}$ Additionally, the MR-PRESSO test can detect and correct for the influence of outliers on MR estimates, ${ }^{24}$ and in MR-Egger regression, the $P$-value of the intercept term can be used as an indicator of directional pleiotropy ( $P$-values less than 0.05 were considered statistically significant) ${ }^{25}$

\section{Results}

\section{Insomnia and fracture risk}

After excluding participants with missing data on the main covariates at baseline ( $n=25,661), 14,005$ out of the 417,853 participants (3.35\%) developed an incident fracture during a median of 7.96 years of follow-up (Supplementary Table 6). In primary analysis (model 0), an association was observed between insomnia and incident fracture, with a 7.0\% higher risk (hazard ratio [HR]: 1.070, 95\% confidence interval [95\% Cl]: 1.045-1.096, $P=1.87 \times 10^{-8}$ ) (Figure 1). Further adjustments for BMD (model 1) and falls (model 2) attenuated the estimated hazard ratios for the association between insomnia and the incidence of 
fracture (HR: $1.066,95 \% \mathrm{Cl}: 1.040-1.091, P=1.90 \times 10^{-7}$ for model 1; HR: $1.044,95 \% \mathrm{Cl}: 1.019-1.069, P=$ $4.19 \times 10^{-4}$ for model 2) (Figure 1). Based on the fully adjusted model (model 3), insomnia was associated with incident fracture, with a $4.0 \%$ higher risk (HR: 1.040, 95\% Cl: 1.015-1.065, $P=0.001$ ) (Figure 1). In addition, we conducted a series of analyses to assess the mediating role of several covariates (i.e., BMD and falls) on the observed associations. Results from the mediation analysis showed that $26.0 \%$ and $10.0 \%$ of the intermediary effect of insomnia on the risk of fracture was mediated by falls and BMD, respectively (Supplementary Table 7 and Supplementary Table 8). These results suggested the mediating effect of falls was larger than BMD in the pathway between insomnia and fracture risk. Findings stratified by sex were consistent with the pooled results in model 0 (HR: $1.092,95 \% \mathrm{Cl}: 1.052-1.135, P=4.79 \times 10^{-6}$ for male; HR: $1.052,95 \% \mathrm{Cl}: 1.020-1.084, P=3.42 \times 10^{-5}$ for female) (Figure 1). Besides, the PAR\% for fracture was estimated as $13.4 \%$, suggesting that $13.4 \%$ of the fracture cases could be removed if insomnia as a risk factor was removed.

In two-sample MR, genetically predicted insomnia was associated with increased risk of fracture (OR: $1.059,95 \% \mathrm{Cl}$ : $1.028-1.090, P=8.97 \times 10^{-5}$ ) with the IVW approach. In the weighted median method, the magnitude of the causal association estimate was similar (Figure 1). MR-Egger regression showed no evidence of directional pleiotropy $(P$ for intercept $=0.711)$. After excluding one outlier, the causal association estimate was consistently using the MR-PRESSO test (OR: 1.056, 95\% Cl: 1.027-1.086, $P=$ $1.94 \times 10^{-4}$ ) (Figure 1). The causality between insomnia and the incident fracture was replicated by onesample MR analysis (HR: 1.077, 95\% Cl: 1.019-1.138, $P=0.008$ ) (Figure 1). Although evidence from twosample MR analyses did not support the association between genetically predicted insomnia and BMD (OR: $1.015,95 \% \mathrm{Cl}: 0.995-1.035, P=0.146$ ), there was a causal relationship between insomnia and risk of falls (OR: $1.014,95 \% \mathrm{Cl}: 1.006-1.022, P=0.001$ ), and to some degree supported the hypothesis that the mediating effect of falls was larger than BMD.

\section{Sleep duration and fracture risk}

In this prospective study, there was evidence of a U-shape association between sleep duration and fracture risk in model 0 ( $P<0.001$ for non-linearity), with the lowest risk of fracture at 7-8 hours per day of sleep duration (Figure 2 and Supplementary Figure 2). Compared to those who slept 7 or 8 hours per night, participants who were short (less than 7 hours of sleep) and long sleepers (more than 8 hours of sleep) had increased risk of fracture in model 0 (HR: $1.171,95 \% \mathrm{Cl}: 1.127$ to $1.217, P=1.19 \times 10^{-15}$; HR: $1.129,95 \% \mathrm{Cl}: 1.062-1.199, P=1.01 \times 10^{-4}$, respectively) (Table 1). We found that the effect of short or long sleep duration on fracture risk was attenuated by additionally adjusting for BMD and falls (model 1 , model 2, and model 3) (Table 1). Falls and BMD were estimated to mediate $20.2 \%$ and $12.9 \%$ of the effect of sleep duration on fracture in observational analyses (Supplementary Table 9 and Supplementary Table 10). The results were consistent in the stratified analysis by sex (Supplementary Table 11). Interestingly, 
compared to participants who slept 7-8 hours per night, short and long sleep duration were estimated to explain a similar percentage (4.48\%) of the population risk of developing a fracture.

In the two-sample MR, we found that genetically determined increased sleep duration was inversely associated with fracture risk (OR: 0.997, 95\% Cl: 0.995-0.999, $P=0.004$ ) (Table 1). After excluding one outlier, similar findings were observed in MR-PRESSO test (OR: 0.997, 95\% Cl: 0.995-0.999, $P=0.009$ ) (Table 1). The evidence from the MR-Egger regression also did not support the presence of directional pleiotropy ( $P=0.229$ for intercept). Furthermore, findings from two-sample MR, which genetically predicted sleep duration was associated with a decreased risk of falls (OR: 0.999, 95\% Cl: 0.999-0.999, $P$ $<0.001$ ), but not with BMD (OR: 1.000, 95\% Cl: 0.998-1.002, $P=0.624$ ).

\section{Snoring, excessive daytime sleepiness, chronotype, and fracture risk}

In the observational study (model 0), we found that snoring was associated with a decreased risk of fracture (HR: 0.947, 95\% Cl: 0.912-0.984, $P=0.005$ ) (Supplementary Table 12). However, while digging into our data, we were aware that participants with snoring were less likely to have insomnia and unnormal sleep duration (Supplementary Table 13). Then, we conducted sensitivity analyses stratified by insomnia symptoms and sleep duration and found that in the participants free of insomnia and with normal sleep duration, and found that the association between snoring and fracture risk was not statistically significant (HR: $0.953,95 \% \mathrm{Cl}: 0.868-1.045, P=0.305)$ (Supplementary Table 12). Similarly, evidence from one-sample MR and two-sample MR did not support this association (OR: $1.214,95 \% \mathrm{Cl}$ : $0.770-1.913, P=0.402$ for two-sample MR; HR: $1.765,95 \% \mathrm{Cl}$ : $0.794-3.921, P=0.163$ for one-sample MR) (Supplementary Table 12). These results suggested that since participants with snoring were more likely in the low-risk group for other sleep factors (i.e., never/rarely had insomnia and had normal sleep duration), the observational estimates may not reflect the causal effects of snoring on fracture risk.

In multivariable Cox regression (model 0), we found statistically significant associations of daytime sleepiness with the risk of fracture (HR: $\left.1.086,95 \% \mathrm{Cl}: 1.052-1.122, P=5.70 \times 10^{-7}\right)$, and being morning preference was a protective factor for fracture risk (HR: 0.963, 95\% Cl: 0.945-0.982, $P=1.13 \times 10^{-4}$ ) (Supplementary Table 14). However, in the two-sample MR, there was no evidence of the association of chronotype (OR: $0.986,95 \% \mathrm{Cl}: 0.954-1.020, P=0.425)$, which was consistent with results from the onesample MR (HR: 0.983, 95\% Cl: 0.921-1.048, $P=0.595)$ (Supplementary Table 14).

\section{The sleep risk score and fracture risk}


Finally, we included four potential fracture-related sleep factors (i.e., insomnia, sleep duration, chronotype, and daytime sleepiness) to develop a sleep risk score (SRS). Each participant received a score of 1 for each of the following sleep behaviors: insomnia ("sometimes" or "usually"), abnormal sleep duration (less than 7 hours per day or more than 8 hours per day), late chronotype ("evening" or "evening than morning"), and frequent daytime sleepiness ("often" or "all the time") (Table 2). All these component scores were summed to generate an SRS, ranging from 0 to 4 . The higher scores indicated poor sleep quality.

By generating the sleep risk score, we assessed the joint effect of sleep behaviors on fracture risk and found that the risk of fracture increased significantly with an increasing SRS (i.e., poor sleep quality) (HR: 1.095, 95\% Cl: 1.073-1.118, $P<2 \times 10^{-16}$ ) (Figure 3). When stratified by sex, the effect estimates of the association between sleep risk score and fracture risk in men were slightly larger than in women (HR: $1.107,95 \% \mathrm{Cl}: 1.071-1.144, P=1.74 \times 10^{-9}$ for male; HR: $1.080,95 \% \mathrm{Cl}: 1.052-1.109, P=8.77 \times 10^{-9}$ for female) (Figure 3). Besides, the PAR\% for fracture was estimated as $19.4 \%$, suggesting that $19.4 \%$ of incident fracture cases in this study would be removed if all participants had been in healthy sleep behaviors.

Based on the baseline characteristics, participants with healthy sleep quality (i.e., lower sleep risk score) have a decreased risk of falls and higher BMD (Table 2). Therefore, to obtain a deeper understanding of the potential mechanisms for the observation, we further included BMD and falls as mediators in the adjusted model. We found that the magnitude of the associations of SRS with fracture risk was attenuated, with wider confidence intervals (HR: $1.058,95 \% \mathrm{Cl}: 1.036-1.080, P=1.07 \times 10^{-7}$ for model 3 )

(Figure 3). the mediation analyses showed that $19.0 \%$ and $11.6 \%$ of the intermediary effect of SRS on the risk of fracture was mediated by falls and BMD (Supplementary Table 15 and Supplementary Table 16).

\section{Discussion}

In this study, using a large-scale UK Biobank dataset, we investigated the association of five sleep behaviors (insomnia, sleep duration, snoring, daytime sleepiness, and chronotype) with fracture risk. Our findings suggest that unhealthy sleep patterns could result in a higher risk of fracture, and $19.4 \%$ of all fracture cases could be removed if all participants exhibited healthy sleep patterns. To some extent, falls had a larger intermediary effect than BMD in the association between sleep and fracture. Both the observational study and MR analyses consistently suggested a causal effect of insomnia on the fracture risk. Further, a U-shape relationship was observed between sleep duration and fracture risk, with the lowest risk of fracture at 7-8 hours per day of sleep duration. 
Previous observational studies examining the associations between insomnia and fracture risk are few, and existing evidence is inconsistent. For example, one study that included 34,163 nursing home residents found no association between insomnia and the risk of hip fracture (OR: 0.99, 95\% Cl: 0.771.26). ${ }^{17}$ However, due to the limited validity of the insomnia Minimum Data Set items used in the analysis, it is possible that their conclusion was prone to bias. ${ }^{26}$ In contrast, results from another cohort study in the Women's Health Initiative (WHI) suggested an association between insomnia and an elevated risk of total fracture (HR: 1.03, 95\% Cl: 1.01-1.06) in multivariable models, ${ }^{13}$ which agreed with our findings. Furthermore, using genetic variants associated with insomnia phenotype, we found strong evidence for the causal effect of insomnia on fracture risk.

Recent epidemiological studies have reported a J-shaped or reverse-J-shaped association between sleep duration and fracture risk, and the evidence is not consistent. For example, a cohort study including 157,306 women found that, compared to subjects sleeping 7 hours per night, short sleepers ( $\leq 5$ hours per night) had a 12\% (95\% Cl: 5\%-20\%) increased odds of all fractures, but no association was found between fracture risk and a long duration of sleep. ${ }^{13}$ In contrast, in another population-based cohort study that included 8,101 women, a long duration of sleep (but not short sleep time) was associated with an increased risk of non-spinal fractures (HR: 1.26, 95\% Cl: $1.00-1.58$ for sleeping $\geq 10$ hours). ${ }^{14}$ In our study, the evidence from observational study suggested a U-shape association between sleep duration and fracture risk. Participants with a sleep duration of 7-8 hours per day had the lowest fracture risk, suggesting that sleeping 7-8 hours per day might the appropriate sleep duration for preventing fracture. However, findings from two-sample MR supported a linear adverse effect of sleep duration (continuous trait as exposure) on the fracture risk. Due to the limited data, ${ }^{27}$ we didn't conduct the MR analyses while taking long/short duration (binary trait) as exposures. We found that, daytime sleepiness was associated with risk of fracture, but being morning preference was a protective factor for fracture risk in observational study. However, our MR analyses found no significant association between genetically determined chronotype and the incidence of fracture, but we cannot preclude weak genetic associations because of the limited statistical power in this study (i.e., 9\%).

Furthermore, for snoring, a cohort study including 3,220 women and 2,699 men found no significant association between snoring with a high fracture risk. However, female participants in the severe snoring group (6-7 nights per week or sleep disturbance by snoring in the next room) had an increased risk of fracture (HR: 1.682, 95\% Cl: 1.164-2.430, P=0.006). ${ }^{28}$ Similarly, a prospective study that included 55,264 women in the Nurses' Health Study (NHS) also found that participants with a history of obstructive sleep apnea, a sleep disorder associated with snoring, ${ }^{29}$ were at a higher risk of vertebral fracture (HR: 1.88 , 95\% Cl: 1.21-2.93). ${ }^{30}$ In our observational study, snoring participants rarely had insomnia and abnormal sleep duration. After excluding participants with insomnia or short/sleep duration, we did not find a 
significant association between snoring and the risk of any fracture. MR analysis results also did not support a causal role of snoring. Taken together, these data suggested that snoring may not be a significant modifiable risk factor for fracture. However, due to the limited data on snoring (e.g., the frequency of snoring), we could not assess the association between severe snoring and fracture risk. Further research is needed to assess the potential dose-response association between snoring severity and any fracture.

Because sleep behaviors might affect with each other, ${ }^{31}$ for example, late chronotype might result in shorter sleep duration and excessive daytime sleepiness. Therefore, based on our results, we constructed an SRS to assess the overall relationship between the combination of sleep behaviors and fracture risk. We found that poor sleep quality was associated with an increased risk of fracture, and $19.4 \%$ of the fracture cases in this population would be removed if all participants exhibited a healthy sleep pattern. These data suggested the importance of careful consideration of sleep patterns, particularly insomnia, in fracture risk assessment and prevention in clinical practice.

Given the associations of sleep disturbance with low bone mineral density (BMD) ${ }^{32}$ and the loss of postural control, ${ }^{33}$ the potential relationship between sleep behavior and fracture risk might be due to the mediating effect of either BMD-related or fall-related factors. In our adjusted models, when including BMD and falls as additional covariables, the magnitude of the associations between sleep behaviors and fracture risk would attenuate, with wider confidence intervals. We observed that a larger proportion of the effect of sleep on fracture risk was mediated by falls than by BMD (e.g., $26 \%$ vs $10 \%$ for insomnia). Also, estimates from MR analyses supported the causal relationship between several sleep behaviors (e.g., insomnia or sleep duration) and the risk of falls. Taken together, these results suggested the stronger mediating role of falls in the association between sleep and fracture risk.

In summary, our observational and Mendelian randomization findings supported an association between poor sleep quality and an increased risk of fracture. The sleep risk score defined by our study (evening preference, sometimes or usually insomnia, abnormal sleep duration, and often or all the time daytime sleepiness) provided a frame of reference for identifying individuals at high risk of fracture. Among the five sleep behaviors, insomnia had a causal effect on fracture risk. Furthermore, fall prevention for reducing fracture risk in individuals with poor sleep quality could be more efficient than improving BMD.

\section{Declarations}

Ethics approval: This study was performed under generic ethical approval obtained by UK Biobank from the National Health Service National Research Ethics Service (approval letter ref 11/NW/0382, 17 June 
2011).

Funding: This work was supported by the National Natural Science Foundation of China (Grants No: 32061143019 and 81871831).

Data availability: No additional data available.

Computer code: The code is available upon request.

Acknowledgments: We thank the GEnetic Factors for OSteoporosis (GEFOS) Consortium and the UK Biobank (Application 41376) for making this work possible. We also thank the High-Performance Computing Center at Westlake University for the facility support and technical assistance.

Author contributors: H.-F.Z. conceptualized and designed the study. Y.Q. and J.X. conducted analysis. Y.Q. drafted the manuscript, P.C., G.-B.C., F.H. and S.K. reviewed and edited manuscript. All authors contributed, discussed and approved manuscript.

Conflict of interest: None declared.

\section{References}

1. Cauley, J. A. Public health impact of osteoporosis. The journals of gerontology. Series A, Biological sciences and medical sciences 68, 1243-1251, doi:10.1093/gerona/glt093 (2013).

2. Johnell, O. \& Kanis, J. A. An estimate of the worldwide prevalence and disability associated with osteoporotic fractures. Osteoporosis international: a journal established as result of cooperation between the European Foundation for Osteoporosis and the National Osteoporosis Foundation of the USA 17, 1726-1733, doi:10.1007/s00198-006-0172-4 (2006).

3. Cooper, C., Campion, G. \& Melton, L. J., 3rd. Hip fractures in the elderly: a world-wide projection. Osteoporosis international: a journal established as result of cooperation between the European Foundation for Osteoporosis and the National Osteoporosis Foundation of the USA 2, 285-289, doi:10.1007/BF01623184 (1992). 
4. Hernlund, E. et al. Osteoporosis in the European Union: medical management, epidemiology and economic burden. A report prepared in collaboration with the International Osteoporosis Foundation (IOF) and the European Federation of Pharmaceutical Industry Associations (EFPIA). Arch Osteoporos 8, 136, doi:10.1007/s11657-013-0136-1 (2013).

5. Trajanoska, K. et al. Assessment of the genetic and clinical determinants of fracture risk: genome wide association and mendelian randomisation study. Bmj 362, k3225, doi:10.1136/bmj.k3225 (2018).

6. Morris, J. A. et al. An atlas of genetic influences on osteoporosis in humans and mice. Nature genetics 51, 258-266, doi:10.1038/s41588-018-0302-x (2019).

7. Estrada, K. et al. Genome-wide meta-analysis identifies 56 bone mineral density loci and reveals 14 loci associated with risk of fracture. Nature genetics 44, 491-501, doi:10.1038/ng.2249 (2012).

8. Kanis, J. A. et al. Smoking and fracture risk: a meta-analysis. Osteoporos Int 16, 155-162, doi:10.1007/s00198-004-1640-3 (2005).

9. Sadeghi, O., Saneei, P., Nasiri, M., Larijani, B. \& Esmaillzadeh, A. Abdominal Obesity and Risk of Hip Fracture: A Systematic Review and Meta-Analysis of Prospective Studies. Adv Nutr 8, 728-738, doi:10.3945/an.117.015545 (2017).

10. Swayambunathan, J. et al. Incidence of Hip Fracture Over 4 Decades in the Framingham Heart Study. JAMA internal medicine, doi:10.1001/jamainternmed.2020.2975 (2020).

11. Xu, C. et al. Circadian Clock Regulates Bone Resorption in Mice. J Bone Miner Res 31, 1344-1355, doi:10.1002/jbmr.2803 (2016).

12. Fu, L., Patel, M. S., Bradley, A., Wagner, E. F. \& Karsenty, G. The molecular clock mediates leptinregulated bone formation. Cell 122, 803-815, doi:10.1016/j.cell.2005.06.028 (2005).

13. Cauley, J. A. et al. Characteristics of Self-Reported Sleep and the Risk of Falls and Fractures: The Women's Health Initiative (WHI). Journal of bone and mineral research: the official journal of the American Society for Bone and Mineral Research 34, 464-474, doi:10.1002/jbmr.3619 (2019).

14. Stone, K. L. et al. Self-reported sleep and nap habits and risk of falls and fractures in older women: the study of osteoporotic fractures. Journal of the American Geriatrics Society 54, 1177-1183, doi:10.1111/j.1532-5415.2006.00818.x (2006).

15. Cauley, J. A. et al. Hypoxia during sleep and the risk of falls and fractures in older men: the Osteoporotic Fractures in Men Sleep Study. J Am Geriatr Soc 62, 1853-1859, doi:10.1111/jgs.13069 (2014).

16. Holmberg, A. H. et al. Risk factors for hip fractures in a middle-aged population: a study of 33,000 men and women. Osteoporos Int 16, 2185-2194, doi:10.1007/s00198-005-2006-1 (2005).

17. Avidan, A. Y. et al. Insomnia and hypnotic use, recorded in the minimum data set, as predictors of falls and hip fractures in Michigan nursing homes. Journal of the American Geriatrics Society 53, 955-962, doi:10.1111/j.1532-5415.2005.53304.x (2005).

18. Smith, G. D. \& Ebrahim, S. 'Mendelian randomization': can genetic epidemiology contribute to understanding environmental determinants of disease? Int J Epidemio/32, 1-22, 
doi:10.1093/ije/dyg070 (2003).

19. Bai, W. Y. et al. Identification of PIEZO1 polymorphisms for human bone mineral density. Bone 133, 115247, doi:10.1016/j.bone.2020.115247 (2020).

20. Xia, J. et al. Systemic evaluation of the relationship between psoriasis, psoriatic arthritis and osteoporosis: observational and Mendelian randomisation study. Ann Rheum Dis 79, 1460-1467, doi:10.1136/annrheumdis-2020-217892 (2020).

21. Chang, C. C. et al. Second-generation PLINK: rising to the challenge of larger and richer datasets. Gigascience 4, 7, doi:10.1186/s13742-015-0047-8 (2015).

22. Burgess, S., Butterworth, A. \& Thompson, S. G. Mendelian randomization analysis with multiple genetic variants using summarized data. Genet Epidemio/37, 658-665, doi:10.1002/gepi.21758 (2013).

23. Bowden, J., Davey Smith, G., Haycock, P. C. \& Burgess, S. Consistent Estimation in Mendelian Randomization with Some Invalid Instruments Using a Weighted Median Estimator. Genet Epidemiol 40, 304-314, doi:10.1002/gepi.21965 (2016).

24. Verbanck, M., Chen, C. Y., Neale, B. \& Do, R. Detection of widespread horizontal pleiotropy in causal relationships inferred from Mendelian randomization between complex traits and diseases. Nature genetics 50, 693-698, doi:10.1038/s41588-018-0099-7 (2018).

25. Bowden, J., Davey Smith, G. \& Burgess, S. Mendelian randomization with invalid instruments: effect estimation and bias detection through Egger regression. Int J Epidemiol 44, 512-525, doi:10.1093/ije/dyv080 (2015).

26. Martin, J. L. \& Alessi, C. A. Limited validity of minimum data set items on sleep and hypnotic use in predicting falls and hip fracture in nursing home residents. J Am Geriatr Soc 54, 1150-1151; author reply 1152-1153, doi:10.1111/j.1532-5415.2006.00777.x (2006).

27. Dashti, H. S. et al. Genome-wide association study identifies genetic loci for self-reported habitual sleep duration supported by accelerometer-derived estimates. Nature communications 10, 1100, doi:10.1038/s41467-019-08917-4 (2019).

28. Choi, S. B., Lyu, I. S., Lee, W. \& Kim, D. W. Increased fragility fracture risk in Korean women who snore: a 10-year population-based prospective cohort study. BMC musculoskeletal disorders 18, 236, doi:10.1186/s12891-017-1587-0 (2017).

29. Mattei, A., Tabbia, G. \& Baldi, S. Diagnosis of sleep apnea. Minerva Med 95, 213-231 (2004).

30. Huang, T., Tworoger, S. S., Redline, S., Curhan, G. C. \& Paik, J. M. Obstructive Sleep Apnea and Risk for Incident Vertebral and Hip Fracture in Women. J Bone Miner Res 35, 2143-2150, doi:10.1002/jbmr.4127 (2020).

31. Fan, M. et al. Sleep patterns, genetic susceptibility, and incident cardiovascular disease: a prospective study of 385292 UK biobank participants. European heart journal 41, 1182-1189, doi:10.1093/eurheartj/ehz849 (2020).

32. Ochs-Balcom, H. M. et al. Short Sleep Is Associated With Low Bone Mineral Density and Osteoporosis in the Women's Health Initiative. Journal of bone and mineral research: the official 
journal of the American Society for Bone and Mineral Research 35, 261-268, doi:10.1002/jbmr.3879 (2020).

33. Robillard, R., Prince, F., Boissonneault, M., Filipini, D. \& Carrier, J. Effects of increased homeostatic sleep pressure on postural control and their modulation by attentional resources. Clinical neurophysiology: official journal of the International Federation of Clinical Neurophysiology 122, 1771-1778, doi:10.1016/j.clinph.2011.02.010 (2011).

\section{Tables}


Table 1 Observational and Mendelian randomization analyses for the relationships of sleep duration with fracture risk.

\begin{tabular}{lccc}
\hline Method & OR/HR & $95 \%$ CI & \begin{tabular}{c}
$P$-value of \\
\cline { 3 - 4 }
\end{tabular} \\
& & association \\
\hline
\end{tabular}

Observational study

Multivariable Cox regression (model 0) sleep 7-8 hours

1 (reference) 1 (reference)

sleep less 7 hours

1.171

$1.127-1.217$

$1.19 \times 10^{-15}$

sleep more than 8 hours

1.129

1.062-1.199

$1.01 \times 10^{-4}$

Multivariable Cox regression (model 1)

sleep 7-8 hours

sleep less 7 hours

sleep more than 8 hours
1 (reference) 1 (reference)

$1.153 \quad 1.109-1.199$

1.116

$1.049-1.187$

$8.21 \times 10^{-13}$

Multivariable Cox regression (model 2)

sleep 7-8 hours

sleep less 7 hours

sleep more than 8 hours
1 (reference) 1 (reference)

1.136

1.099
1.092-1.181

1.034-1.168

$4.75 \times 10^{-4}$

Multivariable Cox regression (model 3)

sleep 7-8 hours

sleep less 7 hours

sleep more than 8 hours
1 (reference) 1 (reference)

1.12

1.086
1.077-1.165

1.021-1.155
$1.35 \times 10^{-10}$

0.002

Two-sample Mendelian randomization

IVW method

0.997

0.995-0.999

0.004

Weighted-median method

0.997

0.995-0.999

0.056

MR-PRESSO Outlier corrected

0.997

0.995-0.999

0.009

MR-Egger regression

0.229

Model 0 was adjusted for confounders, including age, education, sex, smoking, alcohol consumption, physical activity, body mass index, and the use of glucocorticoid; Model $1=$ 
Model $0+$ BMD; Model $2=$ Model $0+$ falls; Model $3=$ Model $0+$ BMD + falls. $*$ the $P-$ value of the intercept term.

Abbreviations: BMD, bone mineral density; CI, confidence interval; $\mathrm{HR}$, hazard ratio; IVW, inverse-variance weighted; MR, Mendelian randomization; MR-PRESSO, MR pleiotropy residual sum and outlier; OR, odds ratio. 
Table 2 Baseline characteristics of participants with different sleep behaviors in prospective studies.

\begin{tabular}{|c|c|c|c|c|c|}
\hline \multirow[t]{2}{*}{ Baseline characteristics } & \multicolumn{5}{|c|}{ Sleep risk score } \\
\hline & 0 & 1 & 2 & 3 & 4 \\
\hline Number of participants & 52040 & 160843 & 116780 & 34074 & 2817 \\
\hline \multicolumn{6}{|l|}{ Having low-risk sleep } \\
\hline \multicolumn{6}{|l|}{ behaviors $^{b}$} \\
\hline \multirow[t]{2}{*}{ Never/rarely insomnia } & 52040 & 28452 & $7400(6.34)$ & $669(1.96)$ & $0(0.00)$ \\
\hline & $(100.00)$ & $(17.69)$ & & & \\
\hline Sleep 7-8 hours per & 52040 & 147437 & 49930 & 3277 & $0(0.00)$ \\
\hline day & $(100.00)$ & $(91.67)$ & $(42.76)$ & $(9.62)$ & \\
\hline \multirow[t]{2}{*}{ Never/rarely dozing } & 52040 & 150619 & 73826 & 6624 & $0(0.00)$ \\
\hline & $(100.00)$ & $(93.64)$ & $(63.22)$ & $(19.44)$ & \\
\hline \multirow[t]{2}{*}{ Morning perference } & 52040 & 156021 & 102404 & 23504 & $0(0.00)$ \\
\hline & $(100.00)$ & $(97.00)$ & $(87.69)$ & (68.98) & \\
\hline \multirow[t]{2}{*}{ Bone mineral density ${ }^{a}$} & $0.56(0.13)$ & 0.54 & 0.54 & 0.54 & 0.54 \\
\hline & & $(0.13)$ & $(0.14)$ & $(0.14)$ & $(0.15)$ \\
\hline \multicolumn{6}{|l|}{ Falls ${ }^{b}$} \\
\hline \multirow[t]{2}{*}{ No } & 45331 & 133315 & 91965 & 24821 & 1911 \\
\hline & $(87.11)$ & (82.89) & $(78.75)$ & $(72.84)$ & $(67.84)$ \\
\hline \multirow[t]{2}{*}{ Yes } & 6709 & 27528 & 24815 & 9253 & 906 \\
\hline & (12.89) & (17.11) & (21.25) & (27.16) & (32.16) \\
\hline \multicolumn{6}{|l|}{ Fracture $^{\mathrm{b}}$} \\
\hline \multirow[t]{2}{*}{ No } & 50627 & 155650 & 112605 & 32710 & 2684 \\
\hline & (97.28) & (96.77) & $(96.42)$ & $(96.00)$ & (95.28) \\
\hline \multirow[t]{2}{*}{ Yes } & $1413(2.72)$ & $5193(3.23)$ & $4175(3.58)$ & 1364 & 133 \\
\hline & & & & $(4.00)$ & $(4.72)$ \\
\hline
\end{tabular}

${ }^{a}$ values are mean (SD); ${ }^{b}$ values are numbers (percentages). 


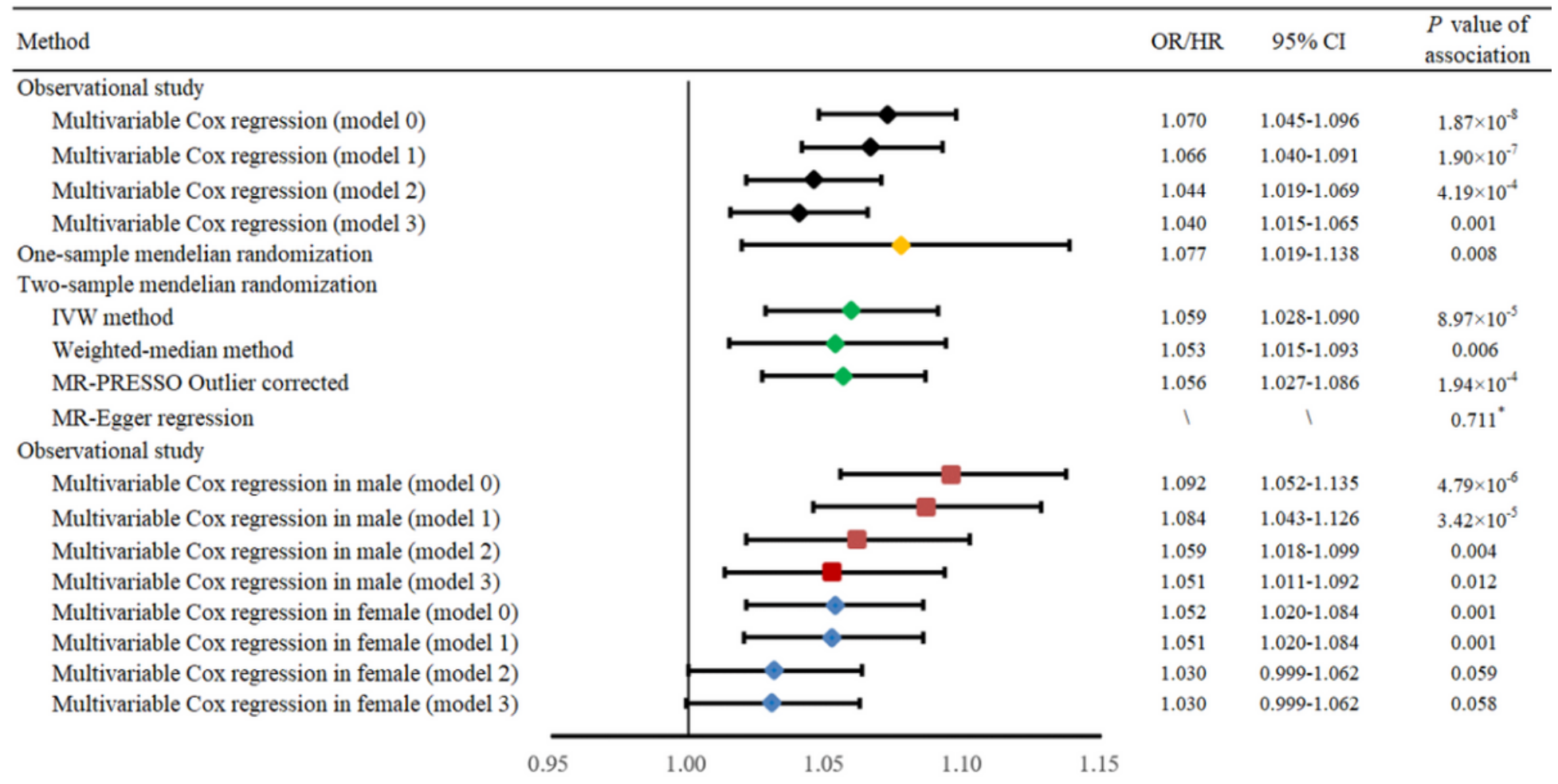

\section{Figure 1}

Forest plot of observational and Mendelian randomization analyses for the relationships of insomnia with fracture risk. Model 1 was adjusted for confounders, including age, education, sex, smoking, alcohol consumption, physical activity, body mass index, and the use of glucocorticoid; Model $1=$ Model $0+$ BMD; Model 2 = Model 0 +falls; Model $3=$ Model 0+ BMD + falls. * the P-value of the intercept term. Abbreviations: $\mathrm{BMD}$, bone mineral density; $\mathrm{Cl}$, confidence interval; $\mathrm{HR}$, hazard ratio; IVW, inverse-variance weighted; MR, Mendelian randomization; MR-PRESSO, MR pleiotropy residual sum and outlier; OR, odds ratio.
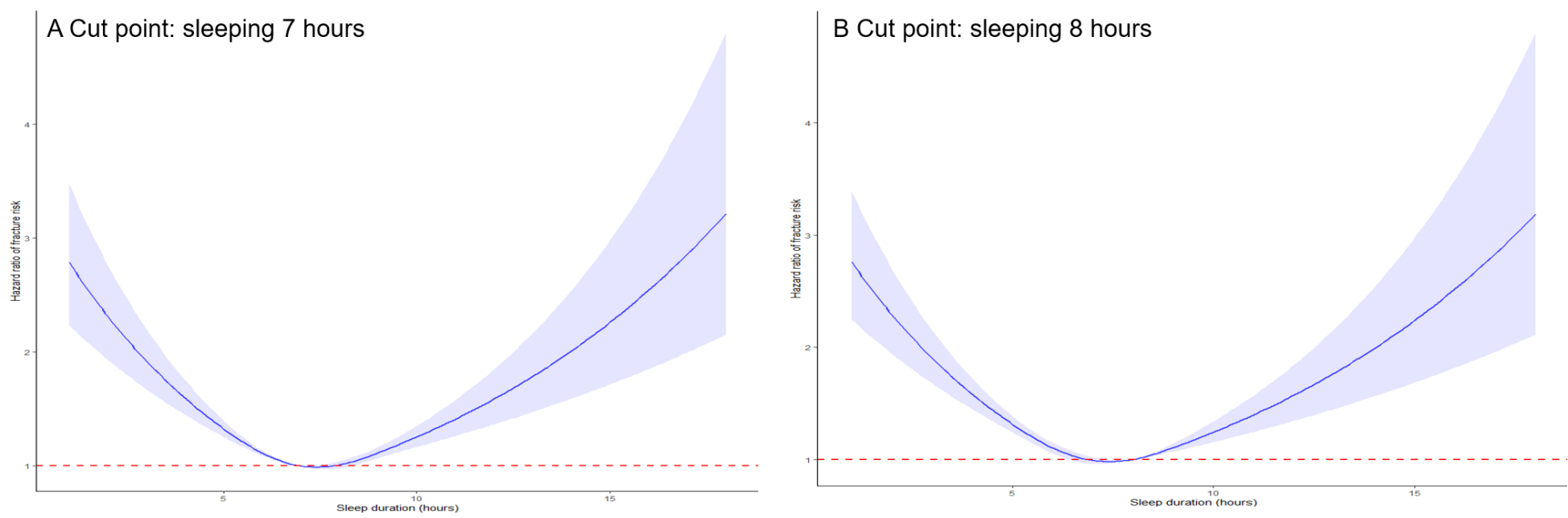

Figure 2 
Observational association of sleep duration with fracture risk using a restricted cubic spline based on model 0 based on different cut points: (A) sleeping 7 hours per day; and (B) sleeping 8 hours per day. Hazard ratios are indicated by solid lines and the $95 \%$ confidence intervals by shaded areas. In all these analyses, models were adjusted for risk factors for fracture, including age, education, sex, smoking, alcohol consumption, physical activity, body mass index, and the use of glucocorticoid.

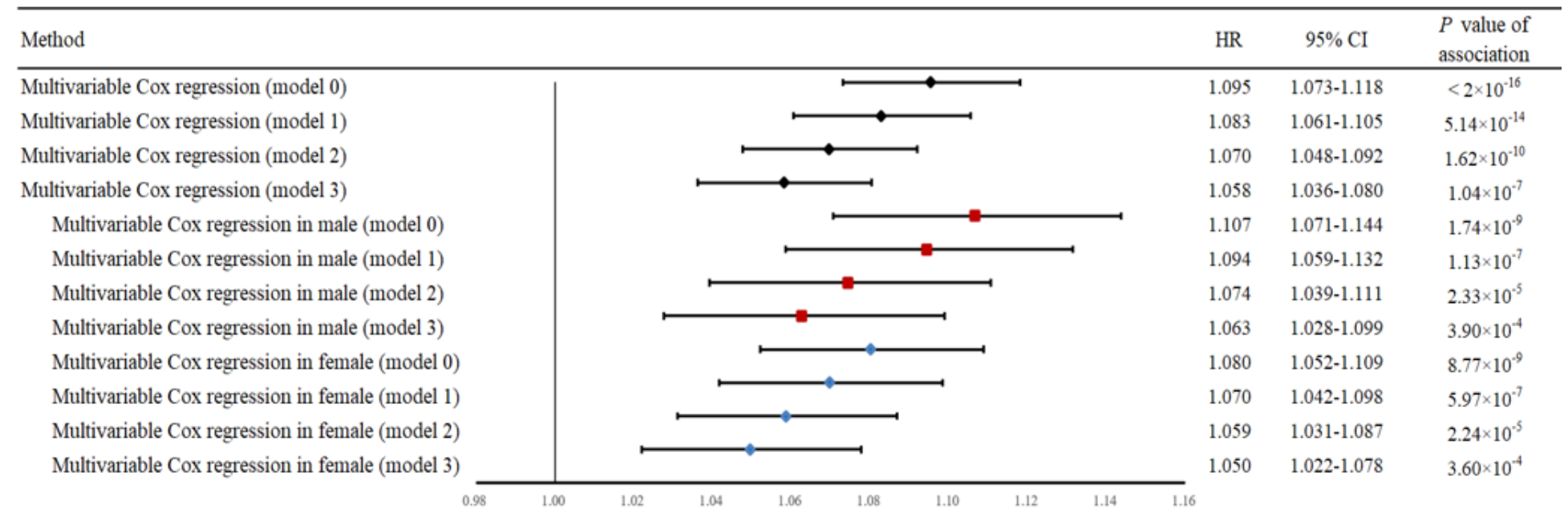

\section{Figure 3}

Forest plot of observational analyses for the relationships of sleep risk score with fracture risk. Model 1 was adjusted for confounders, including age, education, sex, smoking, alcohol consumption, physical activity, body mass index, and the use of glucocorticoid; Model $1=$ Model $0+$ BMD; Model $2=$ Model 0 +falls; Model 3 = Model 0+ BMD + falls. Abbreviations: BMD, bone mineral density; $\mathrm{Cl}$, confidence interval; IVW, inverse-variance weighted; MR, Mendelian randomization; MR-PRESSO, MR pleiotropy residual sum and outlier; OR, odds ratio.

\section{Supplementary Files}

This is a list of supplementary files associated with this preprint. Click to download.

- Supplementarymaterialsv5.docx 